Mit der Revision des Reglements für die FMH-Standeskommission will die FMH gemeinsam mit den Basisorganisationen die Grundregeln vereinheitlichen und die Verfahren beschleunigen. Eine Arbeitsgruppe mit Vertretern der Basisorganisationen und der FMH hat diese Revision, die vom Zentralvorstand genehmigt wurde, realisiert. Zudem hat die Ärztekammer die Standesordnung entsprechend angepasst. Strategisches Ziel der FMH dabei ist die Professionalisierung ihrer Organe.

Dr. med. Jürg Schlup, Präsident der FMH

\title{
Ein neues Reglement für die Standeskommission der FMH
}

\author{
Anne-Sylvie Thiébaud ${ }^{a}$, Michel Meier ${ }^{b}$ \\ a Rechtsanwältin, Abteilung Rechtsdienst FMH; bechtsanwalt, Gesellschaft der Ärztinnen und Ärzte des Kantons Solothurn
}

Im Rahmen der Professionalisierung der FMH-Organe wurde das Reglement der Standeskommission der FMH revidiert. Diese Revision war hauptsächlich darauf ausgerichtet, die allgemeinen Verfahrensbestimmungen noch weiter zu vereinheitlichen, das Verfahren zu vereinfachen und zu beschleunigen. Ausserdem diente die Revision dazu, den Basisorganisationen ein Musterverfahrensreglement zur Verfügung zu stellen.

\section{Einleitung}

Die Standesordnung der FMH (StaO) und das Reglement der Standeskommission der FMH (SK FMH) sind 1997 in Kraft getreten. In der Praxis werden die betreffenden Bestimmungen von den Standeskommissionen der Basisorganisationen (kantonale Ärztegesellschaften, VSAO und VLSS - erste Instanzen) sowie von der SK FMH (zweite Instanz) angewandt. Diese legen den Inhalt der Bestimmungen aus und sorgen dafür, dass die Standesordnung eingehalten wird. Die Standesordnung regelt nicht nur die Beziehungen zwischen dem Arzt und seinen Patienten und Berufskollegen, sondern auch sein Verhalten gegenüber der Gesellschaft und seinen Partnern im Gesundheitswesen.

Im Jahr 2013 ist die Abteilung Rechtsdienst der FMH mit den kantonalen Standeskommissionen zusammengekommen, um eine Zwischenbilanz zu ziehen. Dabei wurde festgehalten, dass die Verfahrensregeln für die Anwendung der StaO klarer festgelegt werden müssen und dass eine Datenbank mit anonymisierten Entscheiden der Standeskommissionen eingerichtet werden muss. Auch die Schaffung eines Musterverfahrensreglements fand grossen Anklang.

Eine der Massnahmen, die im Rahmen der Strategie 2013-2016 der FMH vorgesehen sind, besteht darin, einen Beitrag zur Professionalisierung der Standeskommissionen in den Basisorganisationen zu leisten. Deshalb genehmigte der Zentralvorstand der FMH im Januar 2014 die Bildung einer Arbeitsgruppe, welche die folgenden Aufgaben hatte: Vorschlagen von Änderungen im Reglement der SK FMH, Erarbeitung eines Musterverfahrensreglements für die Basisorganisationen und Einrichtung einer Datenbank mit anonymisierten Entscheiden der Standeskommissionen (erste und zweite Instanzen). Die Arbeitsgruppe «Standesordnung» stand unter der Leitung von Dr. med. Jürg Schlup und umfasste im Weiteren die folgenden Mitglieder: Dr. med. Marianne Winterhalter (ehemalige Präsidentin der Standeskommission des Kantons Basel-Stadt), Dr. med. René Raggenbass (Präsident der Standeskommission des Kantons Wallis), Dr. med. Urs Strebel (ehemaliger Vizepräsident der Standeskommission der FMH), Michel Meier (Rechtsanwalt der Gesellschaft der Ärztinnen und Ärzte des Kantons Solothurn und juristischer Sekretär der kantonalen Standeskommission) sowie Anne-Sylvie Thiébaud (Rechtsanwältin in der Abteilung Rechtsdienst der FMH und zuständig für den Bereich Standesrecht).

\section{Ansatz und Ziele der Revision}

Die beauftragte Arbeitsgruppe erarbeitete ein neues Verfahrensreglement für die Standeskommission der FMH und ein Musterverfahrensreglement, welches den Basisorganisationen zur Verfügung stehen soll. Die Basisorganisationen und die Standeskommissionen wurden zwei Mal konsultiert, zum ersten Mal im Herbst 2014 und zum zweiten Mal Anfang 2015. 
Im Anschluss an die Konsultationen der Basisorganisationen konnten unter Berücksichtigung der Praxis die wichtigsten Ziele umgesetzt werden, d.h. zum einen eine weitergehende Vereinheitlichung der Grundregeln und zum anderen die Vereinfachung und Beschleunigung des Verfahrens. Zahlreiche dieser Bestimmungen, d.h. die Artikel 4 bis 27 des neuen Reglements der SK FMH, gelten nicht nur vor der SK FMH, sondern auch vor den kantonalen Standeskommissionen. So ist in Artikel 43 Absatz 6 der StaO Folgendes festgelegt: «Die allgemeinen Verfahrensbestimmungen im Reglement der Standeskommission der FMH gelten auch im Verfahren vor der Standeskommission der kantonalen Ärztegesellschaft, des VSAO und des VLSS.»

Hinsichtlich der Realisierung dieser Ziele lassen sich die wichtigsten Änderungen im Reglement für die SK FMH wie folgt zusammenfassen:

- Zuständigkeit der Standeskommission wird durch den Wechsel der Basisorganisation oder bei der Beendigung der Mitgliedschaft nicht berührt (Art. 5)

- Um das Schlichtungsverfahren weiter zu fördern, ist in der Regel ein erstinstanzlichen Verfahren in zwei Stufen vorgesehen (Art. 6)

- Präzisierung der Regeln für den Anzeiger und Kläger (Art. 7) sowie derer für Patienten mit Parteistellung (Art. 8)

- Unterbrechung der Verjährung (Art. 10)

- Klarstellung in Bezug auf die Verfahrenssprache (Art. 11)

- Nichtberücksichtigung der Gerichtsferien und nur ein Schriftenwechsel (Art. 21), Möglichkeit, den Entscheid nur im Dispositiv zu eröffnen (Art. 25)

- Vereinheitlichung und Schaffung einer Grundlage für die Verfahrenskosten (Art. 23)

- Aufbau einer Datenbank und Eingabe der Entscheide in diese Datenbank (Art. 27)

Was die Regeln anbelangt, die nur vor der Beschwerdeinstanz (SK FMH) gelten, ist auf zwei wesentliche Änderungen hinzuweisen:

- Änderung und Klarstellung in Bezug auf den Inhalt und die Form der Beschwerde (Art. 32)

- Abschaffung des Schlichtungsversuchs, wobei die Möglichkeit vorgesehen wird, bei Bedarf eine mündliche Verhandlung abzuhalten (Art. 34)

Im Juni 2015 hat der Zentralvorstand der FMH die Revision des Reglements der SK FMH genehmigt, dass das Musterverfahrensreglement den Basisorganisationen zur Verfügung gestellt wird. Das neue Reglement ist am 29. August 2015 in Kraft getreten; die Dokumente sind auf der Website der FMH verfügbar [1].

\section{Änderung von Artikel 44 der Standes- ordnung der FMH}

Parallel dazu hat die Ärztekammer im Rahmen dieser Revision den Artikel 44 der Standesordnung der FMH am 7. Mai 2015 geändert [2]. Falls das Reglement der SK FMH oder der Basisorganisationen eine Regelungslücke aufweist, gelten künftig die Bestimmungen der Schweizerischen Zivilprozessordnung (ZPO) statt das Bundesgesetz über das Verwaltungsverfahren. Das Zivilverfahren kommt dem Verfahren, das vor den Standeskommissionen zur Anwendung gelangt, am nächsten. Die Standeskommissionen haben einen besonderen Status: Sie sind weder Strafbehörden noch zivile Behörden und auch keine Verwaltungsbehörden. Trotzdem sprechen sie Disziplinarmassnahmen aus. In Artikel 44 der StaO FMH wurde auf die Bestimmungen des Bundesgesetzes über das Verwaltungsverfahren verwiesen. Dieser Verweis hatte historische Gründe, denn das Zivilverfahren war in der Schweiz nicht einheitlich geregelt. Die ZPO ist 2011 für die ganze Schweiz in Kraft getreten. Sie wird künftig auf Verfahren vor den Standeskommissionen angewandt, wenn eine Frage in der StaO oder im Verfahrensreglement nicht geregelt ist. Wenn das Protokoll der Ärztekammer nicht angefochten wird, tritt der geänderte Artikel $44 \mathrm{StaO}$ am 29. August 2015 in Kraft.

\section{Fazit}

Die neuen Hilfsmittel, die den kantonalen Standeskommissionen und der SK FMH zur Verfügung stehen, sind weder vollständig noch abschliessend. Doch sie sollten zu einer Professionalisierung dieser Organe und zu einer einheitlicheren Anwendung der StaO beitragen. Die künftige Datenbank mit den anonymisierten Entscheiden der Standeskommissionen wird voraussichtlich im Frühjahr 2016 einsatzbereit sein und dann die oben erwähnten Hilfsmittel ergänzen; sie wird ausschliesslich den Standeskommissionen zur Verfügung stehen. Dank der Zusammenarbeit innerhalb der interdisziplinären Arbeitsgruppe, die sich aus Ärzten und Juristen der ersten und der zweiten Instanz zusammensetzte, und dank der Konsultation der Basisorganisationen konnten mehrere Regelungslücken und Probleme besprochen werden, die bei der Anwendung der StaO aufgetreten sind. Auf dieser Grundlage konnten auch Lösungen umgesetzt werden, die - soweit als möglich und unter Berücksichtigung der allgemeinen Rechtsgrundsätze - pragmatisch sein sollen.

Referenzen

1 www.fmh.ch/ueber_fmh/rechtliche_grundlagen/standesord nung.html

2 Henzen M. Protokoll der ersten Ärztekammer im Jahr 2015. Schweiz Ärztezeitung. 2015;96(27-28):992-1015. 